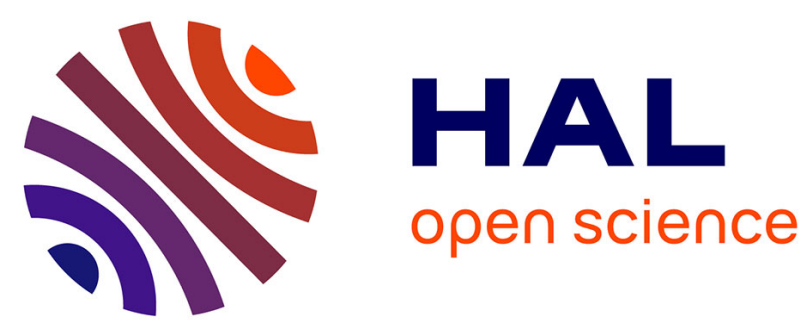

\title{
Estimation of Contact Forces and Floating Base Kinematics of a Humanoid Robot Using Only Inertial Measurement Units
}

\author{
Alexis Mifsud, Mehdi Benallegue, Florent Lamiraux
}

\section{- To cite this version:}

Alexis Mifsud, Mehdi Benallegue, Florent Lamiraux. Estimation of Contact Forces and Floating Base Kinematics of a Humanoid Robot Using Only Inertial Measurement Units. IEEE/RSJ International Conference on Intelligent Robots and Systems (IROS 2015), Sep 2015, Hamburg, Germany. 6p. hal01142399v2

\section{HAL Id: hal-01142399 \\ https://hal.science/hal-01142399v2}

Submitted on 29 Jul 2015

HAL is a multi-disciplinary open access archive for the deposit and dissemination of scientific research documents, whether they are published or not. The documents may come from teaching and research institutions in France or abroad, or from public or private research centers.
L'archive ouverte pluridisciplinaire HAL, est destinée au dépôt et à la diffusion de documents scientifiques de niveau recherche, publiés ou non, émanant des établissements d'enseignement et de recherche français ou étrangers, des laboratoires publics ou privés. 


\title{
Estimation of Contact Forces and Floating Base Kinematics of a Humanoid Robot Using Only Inertial Measurement Units
}

\author{
Alexis Mifsud $^{1,2}$, Mehdi Benallegue ${ }^{1,2}$ and Florent Lamiraux ${ }^{1,2}$
}

\begin{abstract}
A humanoid robot is underactuated and only relies on contacts with environment to move in the space. The ability to measure contact forces and torques enables then to predict the robot dynamics including balance. In classical cases, a humanoid robot is considered as a multi-body system with rigid limbs and joints and interactions with the environment are modeled as stiff contacts. Forces and torques at contacts are generally estimated with sensors which are expensive and sensitive to calibration errors. However, a robot is not perfectly rigid and contacts may have flexibilities. Therefore, external forces create geometric deformations of the body or its environment. These deformations may modify the robot dynamics and produce unwanted and unbalanced motions. Nonetheless, if we have a model of contact stiffness and are able to reconstruct reliably the geometric deformation, we can reconstruct forces and torques at contact. This study aims at estimating contact forces and torques and to observe the body kinematics of the robot with only an Inertial Measurements Unit (IMU). We show that we are able to reconstruct efficiently the position of the Center of Pressure (CoP) of the robot with only the IMU and proprioceptive data from the robot.
\end{abstract}

\section{Problem Statement}

The body dynamics of a legged robot depends mostly on the wrench (forces and moments) applied on contact points. One important illustration is the problem of maintaining balance for a humanoid robot. During locomotion and all other standing tasks, the robot relies entirely on contacts to generate the forces and torques which compensate for gravity and generate a desired trajectory [1]. Therefore, most solutions to control and to balance a humanoid robot generate reference contact forces/torques either directly [2], [3] or by using a related variable such as the center of pressure (CoP) [4]).

Usually robots are considered rigid and the interactions with environment are modeled as stiff contacts. This modeling is convenient in numerical point of view and allows simpler development and prediction of the dynamics. Beside the fact that the environment is not always stiff, several robots do have flexible parts. The flexibility may be in the sole of the feet, but also inside the kinematics tree. An example is the robot HRP-2 which has a flexible bush between the foot and the ankle intended to absorb impacts [5] (see Fig. 1).

\footnotetext{
*This work is supported by the project ERC Advanced Grant 340050 Actanthrope

*This work was partially funded by the French Romeo-2 project

Alexis Mifsud (alexis.mifsudegmail.com), Mehdi Benallegue (mehdi@benallegue.com) and Florent Lamiraux (florentelaas.fr) are with:

1 CNRS, LAAS, 7 avenue du colonel Roche, F-31400 Toulouse, France

${ }^{2}$ Univ de Toulouse, LAAS, F-31400 Toulouse, France
}

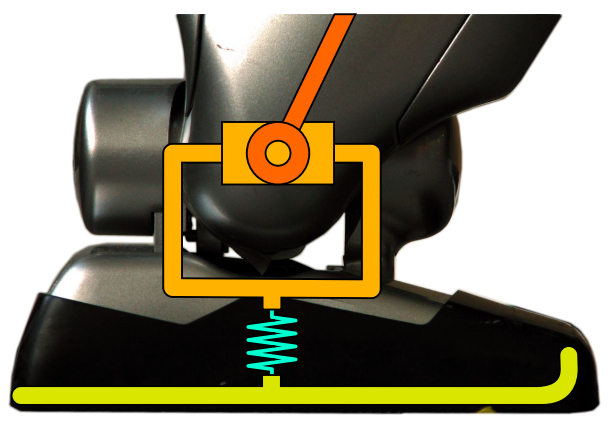

Fig. 1. The foot of HRP-2. Between the ankle joint and the sole of the robot, there is a rubber bush.

As a foot applies forces and torques on the environment, the reactive wrench applies on the foot and causes deformation of the flexible parts. This deformation has two consequences: (i) it modifies the position of all the robot in the world frame, including the end effectors, interfering with environment-related tasks, and (ii) changes the dynamics of the body including the center of mass $(\mathrm{CoM})$ and therefore affects balance.

Nevertheless HRP-2 is able to walk and maintain balance, because there is a stabilizer module which compensates for the flexibility. The current stabilizer uses force/torque sensor measurements and a model of the elasticity of flexibility to track the reference contact wrench [6], [7]. However, the use of these sensors requires a fine calibration which may deteriorate during operation subject to constraints and impacts. Furthermore, these sensors are expensive and some robots are not equipped with them such as Aldebaran's Romeo robot [8].

On the other hand, if we have a model of the elasticity of the flexible parts of the robot, we are able to link their deformation to applied forces and torques. This means that if we can reconstruct accurately the deformation, we may estimate contact forces. This deformation may be seen as a modification of the kinematics of the robot which can be reconstructed using only kinematic sensors such as inertial measurement units (IMUs). These sensors are cheap, relatively reliable and less sensitive to impacts and environmental effects. They provide not only the gravitational field direction, but also measurements on the deviation between the robot local frame and the inertial frame (acceleration and angular velocities).

Moreover, we have shown previously that when we couple the measurements of these sensors to information of contact positions, we can reconstruct the deformation in real-time and with high accuracy [9]. Contact information provides observability and improves accuracy of the observation. We show in this paper that 
by using only these measurements, and by adding a model of the elasticity we are able not only to reconstruct accurate second order kinematics for a humanoid robot, but also to estimate contact forces and moments.

In Section II we describe our elasticity model and its development to dynamics. We show then in Section III that our model provides observability to floating base kinematics and to contact wrenches with only IMU measurements and contact information. This section ends with the description of the estimator before showing in Section IV experimental setting and results which validate the model and the observer.

\section{MODELING}

\section{A. Model of flexibility and state vector}

HRP-2 is a joint-position controlled robot, and as many robots, it has very stiff actuation. Its only non-rigid part is the flexible bush between the ankle joint and the sole. We may consider that the mass of the sole is negligible compared to the total mass of the robot. That means that in a local reference frame, all the limb positions are perfectly known at each instant, and thus the center of mass (CoM) also. We denote this local frame, also called control frame, by $\mathcal{R}_{l}$. In this paper the subscript $l$ for variables stands for local frame and means that they are expressed in $\mathcal{R}_{l}$. The absence of subscript means that the variable is expressed in the world reference frame $\mathcal{R}$.

We consider then the robot as a moving but rigid multi-body system which is connected to its environment through non-stiff contacts. Each body $\mathcal{B}_{i}$ of mass $m_{i}$ has a position $\mathbf{c}_{l, i}$ and orientation matrix $R_{l, i}$ in the control frame $\mathcal{R}_{l}$ (see fig. 2). This position is represented by the homogeneous matrix:

$$
M_{l}^{\mathcal{B}_{i}}=\left(\begin{array}{cc}
R_{l, i} & \mathbf{c}_{l, i} \\
000 & 1
\end{array}\right)
$$

The robot being stiff in the control frame, the flexibility deformation applies on the robot a rigid transformation from control frame $\mathcal{R}_{l}$ to the world reference frame $\mathcal{R}$. It can be then represented by a homogeneous matrix

$$
M_{\text {flex }}=\left(\begin{array}{cc}
R & \mathbf{t} \\
000 & 1
\end{array}\right)
$$

Indeed, due to flexibility, the position of each body $\mathcal{B}_{i}$ is modified and can be represented by an equivalent homogeneous matrix in the world reference frame $\mathcal{R}$ (see fig. 2).

$$
M^{\mathcal{B}_{i}}=\left(\begin{array}{cc}
R_{i} & \mathbf{c}_{i} \\
000 & 1
\end{array}\right)=M_{\text {flex }} M_{l}^{\mathcal{B}_{i}}
$$

Therefore, the flexibility deformation may be represented by its rotation matrix $R$ and its translation $\mathbf{t}$. To model our system, we need to describe the second order dynamics of the flexibility. We choose then to represent the flexibility state vector as:

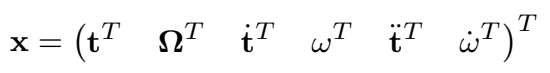

where $\boldsymbol{\Omega}$ is defined in such way that $R=\exp \left([\boldsymbol{\Omega}]_{\times}\right)$with $[\cdot]_{\times}$the skew-symmetric operator such that:

$$
\left[\begin{array}{l}
x \\
y \\
z
\end{array}\right]_{\times}=\left[\begin{array}{ccc}
0 & -z & y \\
z & 0 & -x \\
-y & x & 0
\end{array}\right]
$$

We present in this section a relation between the current state and the induced contact wrench, and using this wrench we model

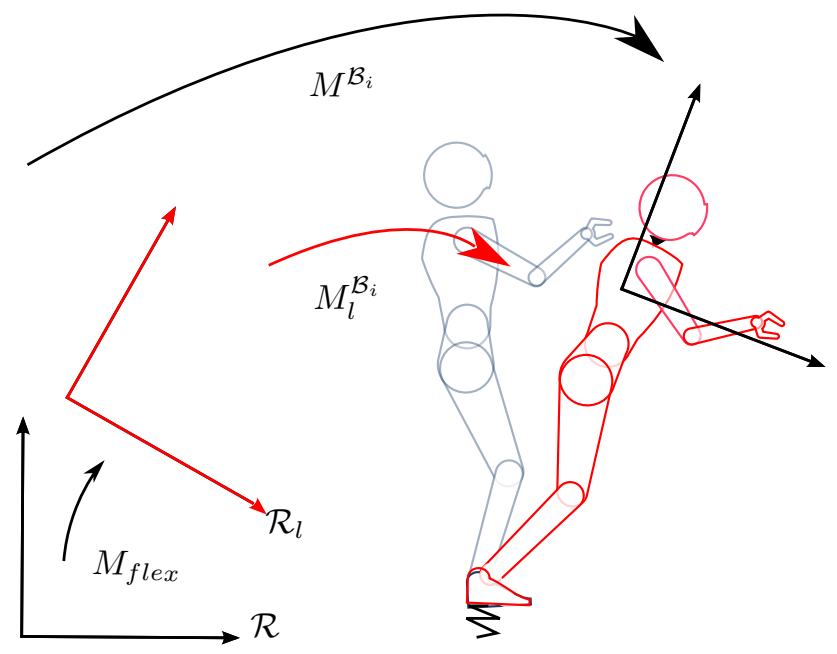

Fig. 2. Definition of the frames used for the modeling of the dynamics of the flexibility. $M_{l}^{\mathcal{B}_{i}}$ is the position of the body $\mathcal{B}_{i}$ in the rigid-body control frame $\mathcal{R}_{l} . M^{\mathcal{B}_{i}}$ is the position of the same body $\mathcal{B}_{i}$ in the world reference frame $\mathcal{R}$. $M_{\text {flex }}$ is the homogeneous matrix between these two frames.

the dynamics of the state vector. It finally enables us to find a model of the flexibility state dynamics in order to predict its evolution using only values expressed in $\mathcal{R}_{l}$.

\section{B. Model of contact wrenches}

The humanoid robot is always in contact with its environment. These contacts can be detected by simple and robust sensors such as pressure sensors [10]. They can also be planned by an external motion planner.

We model the elasticity of each contact as a translational and rotational $6 \mathrm{D}$ damped spring. This gives us a relation between the contact force $\mathbf{F}_{c}$, moment $\mathbf{T}_{c}$, the flexibility state $\mathbf{x}$ and the contact positions in the control frame $\mathcal{R}_{l}$.

1) Forces: We consider that the contacts are firmly connected to environment, the forces are then proportional to the distance $\mathbf{t}_{c i}$ between the current position $\mathbf{p}_{c i}$ of the contact and its position $\mathbf{p}_{c i, l}$ at rest state of the elasticity (zero deformation and zero velocity).

Therefore, for the contact $i$, the relation between the force $\mathbf{F}_{c i}$ generated by the translational spring, with positive definite matrices for stiffness $K_{F s}$ and damping $K_{F d}$, is the following:

$$
\mathbf{F}_{c i}=-K_{F s} \mathbf{t}_{c i}-K_{F d} \dot{\mathbf{t}}_{c i}
$$

where

$$
\mathbf{t}_{c i}=R \mathbf{p}_{c i, l}+\mathbf{t}-\mathbf{p}_{c i, l}
$$

with

$$
\mathbf{p}_{c i}=R \mathbf{p}_{c i, l}+\mathbf{t}
$$

By derivation of (7) and having $\dot{\mathbf{p}}_{c i, l}=\mathbf{0}$ we obtain:

$$
\dot{\mathbf{t}}_{c i}=[\omega]_{\times} R \mathbf{p}_{c i, l}+\dot{\mathbf{t}}
$$

We finally sum the forces $\mathbf{F}_{c i}$ for the number of contacts $n_{c}$. We obtain an expression giving us the total external contact forces:

$$
\begin{aligned}
\mathbf{F}_{c}=\sum_{i=1}^{n_{c}}\left(-K_{F s}\right. & \left(R \mathbf{p}_{c i, l}+\mathbf{t}-\mathbf{p}_{c i, l}\right) \\
- & \left.K_{F d}\left([\omega]_{\times} R \mathbf{p}_{c i, l}+\dot{\mathbf{t}}\right)\right)
\end{aligned}
$$


2) Moments: To simplify computations, all the dynamics of the robot is expressed at the origin of the world reference frame $\mathcal{R}$. Therefore the moment generated by a contact $i$ is composed by the torque of the rotational spring characterized by the stiffness $K_{T s}$ and damping $K_{T d}$, also positive definite matrices, summed to the moment of the contact force $\mathbf{F}_{c i}$. It expression is the following:

$$
\mathbf{T}_{c i}=-K_{T s} \boldsymbol{\Omega}-K_{F d} \omega+\left[\mathbf{p}_{c i}\right]_{\times} \mathbf{F}_{c i}
$$

where $\mathbf{p}_{c i}=R \mathbf{p}_{c i, l}+\mathbf{t}$ is the contact position in $\mathcal{R}$ and $\left[\mathbf{p}_{c i}\right]_{\times} \mathbf{F}_{c i}$ is the moment of the contact force $\mathbf{F}_{c i}$. We finally sum the torques $\mathbf{T}_{c i}$ over the $n_{c}$ contacts and we obtain the total external moments:

$$
\mathbf{T}_{c}=\sum_{i=1}^{n_{c}}\left(-K_{T s} \boldsymbol{\Omega}-K_{F d} \omega+\left[R \mathbf{p}_{c i, l}+\mathbf{t}\right]_{\times} \mathbf{F}_{c i}\right)
$$

\section{Model of the flexibility state dynamics}

We want to model the influence of external contact forces $\mathbf{F}_{c}$ and torques $\mathbf{T}_{c}$ on the flexibility state. These equations provide a prediction for the dynamics of the flexibility as soon as we have reliable informations about internal dynamic variable. These parameters are all required in the local reference frame and are namely the mass, the position, velocity and acceleration of the $\mathrm{CoM}$, the tensor of inertia and its derivative, and the total angular momentum and its derivative. We describe here how the predictor expressions are obtained.

Newton-Euler equations give us a relation between external forces $\mathbf{F}$ and torques $\mathbf{T}$ and the variation of linear and angular momenta of the robot.

1) Newton equation: According to previous section notations, and the multi-body nature of the robot, Newton equation gives us the following relation:

$$
\mathbf{F}=\frac{d}{d t}\left(\sum_{i=1}^{n} m_{i} \dot{\mathbf{c}}_{i}\right)
$$

The right side of this equation corresponds to the derivative of the linear momentum due to the motions of the centers of mass of each body. This can be simplified using the Newton equation, reducing it to the center of mass of the robot:

$$
\mathbf{F}=\frac{d}{d t}(m \dot{\mathbf{c}})
$$

By expressing this linear momentum in $\mathcal{R}_{l}$ using (3) for the CoM position and using two time-derivations, we obtain:

$$
\mathbf{F}=m\left([\dot{\omega}]_{\times} R \mathbf{c}_{l}+[\omega]_{\times}^{2} R \mathbf{c}_{l}+2[\omega]_{\times} R \dot{\mathbf{c}}_{l}+R \ddot{\mathbf{c}}_{l}+\ddot{\mathbf{t}}\right)
$$

If we distinguish in $\mathbf{F}$ the contact forces $\mathbf{F}_{c}$ from the weight we obtain the following equation giving the flexibility dynamics induced by contact forces $\mathbf{F}_{c}$ :

$$
\begin{aligned}
\mathbf{F}_{c}= & {[\dot{\omega}]_{\times} R m \mathbf{c}_{l}+[\omega]_{\times}^{2} R m \mathbf{c}_{l}+2[\omega]_{\times} R m \dot{\mathbf{c}}_{l} } \\
& +R m \ddot{\mathbf{c}}_{l}+m \ddot{\mathbf{t}}+g m \mathbf{u}_{z}
\end{aligned}
$$

with $\mathbf{u}_{z}=\left[\begin{array}{lll}0 & 0 & 1\end{array}\right]^{T}$ the unit vector along the vertical $\mathbf{z}$ axis.

2) Euler equation: The sum of external moments is equal to time-variation of the total angular momentum $\sigma$ of the body. Each body $\mathcal{B}_{i}$ which has an inertia tensor $I_{i}$, an orientation $R_{i}$ and an angular velocity $\omega_{i}$ in $\mathcal{R}$ has a contribution on the total angular momentum. These angular momenta are summed in the following relation:

$$
\mathbf{T}=\dot{\sigma}=\frac{d}{d t} \sum_{i=1}^{n}\left(R_{i} I_{i} R_{i}^{T} \omega_{i}+m_{i}\left[\mathbf{c}_{i}\right]_{\times} \dot{\mathbf{c}}_{i}\right)
$$

For each body $\mathcal{B}_{i}$ in this equation we can distinguish between the angular momentum due to the angular velocity of the body in $\mathcal{R}$ and the one due to its translational velocity in $\mathcal{R}$. By introducing (3) in (17) we can also distinguish between $\sigma_{l}, \sigma_{\omega}$ and $\sigma_{\mathbf{t}}$ such that:

$$
\sigma=R \sigma_{l}+\sigma_{\omega}+\sigma_{\mathbf{t}}
$$

with

$$
\sigma_{l}=\sum_{i=1}^{n}\left(R_{l, i} I_{i} R_{l, i}^{T} \omega_{l, i}+m_{i}\left[\mathbf{c}_{l, i}\right]_{\times} \dot{\mathbf{c}}_{l, i}\right)
$$

the angular momentum of the multi-body in $\mathcal{R}_{l}$ containing only quantities expressed in $\mathcal{R}_{l}$ which have to be expressed in $\mathcal{R}$. We also have the angular momentum due to the angular velocity of the flexibility:

$$
\sigma_{\omega}=R I_{l} R^{T} \omega
$$

where $I_{l}=\sum_{i=1}^{n} R_{l, i} I_{i} R_{l, i}^{T}-\sum_{i=1}^{n} m_{i}\left[\mathbf{c}_{l, i}\right]_{\times}^{2}$ is the total tensor of inertia of the multi-body expressed in the local frame $\mathcal{R}_{l}$. Finally we have:

$$
\sigma_{\mathbf{t}}=m\left[R \mathbf{c}_{l}\right]_{\times} \dot{\mathbf{t}}+m[\mathbf{t}]_{\times} \frac{d}{d t}\left(R \mathbf{c}_{l}+\mathbf{t}\right)
$$

representing the angular momentum due to the linear velocity of the flexibility. After derivation we obtain the following Euler equation:

$$
\begin{aligned}
\mathbf{T}= & {[\omega]_{\times} R I_{l} R^{T} \omega+R \dot{I}_{l} R^{T} \omega+R I_{l} R^{T} \dot{\omega}+[\omega]_{\times} R \sigma_{l} } \\
& +R \dot{\sigma}_{l}+[\mathbf{t}]_{\times} \mathbf{F}+m\left[R \mathbf{c}_{l}\right]_{\times} \ddot{\mathbf{t}}
\end{aligned}
$$

If we distinguish the contact torques $\mathbf{T}_{c}$ from the moment of the weight we have then:

$$
\begin{aligned}
\mathbf{T}_{c}= & {[\omega]_{\times} R I_{l} R^{T} \omega+R \dot{I}_{l} R^{T} \omega+R I_{l} R^{T} \dot{\omega}+[\omega]_{\times} R \sigma_{l} } \\
& +R \dot{\sigma}_{l}+[\mathbf{t}]_{\times} \mathbf{F}_{c}+m\left[R \mathbf{c}_{l}\right]_{\times} \ddot{\mathbf{t}}+\left[R \mathbf{c}_{l}\right]_{\times} g m \mathbf{u}_{z}
\end{aligned}
$$

3) Model of the flexibility state dynamics: By decoupling and inverting the system $(16,23)$ we finally get the following flexibility dynamics:

$$
\begin{aligned}
\dot{\omega}= & R\left(I_{l}+m\left[\mathbf{c}_{l}\right]_{\times}^{2}\right)^{-1} R^{T} \\
& \left(\mathbf{T}_{c}-\left(\left([\omega]_{\times} R I_{l} R^{T}+R \dot{I}_{l} R^{T}\right) \omega+R \dot{\sigma}_{l}+[\omega]_{\times} R \sigma_{l}\right)\right. \\
& +\left[R \mathbf{c}_{l}\right]_{\times}\left(R m \ddot{\mathbf{c}}_{l}+2 m[\omega]_{\times} R \dot{\mathbf{c}}_{l}+m[\omega]_{\times}^{2} R \mathbf{c}_{l}\right) \\
& \left.-\left[R \mathbf{c}_{l}+\mathbf{t}\right]_{\times} \mathbf{F}_{c}\right)
\end{aligned}
$$

and:

$$
\begin{aligned}
\ddot{\mathbf{t}}= & \frac{1}{m}\left(\mathbf{F}_{c}-\left(R m \ddot{\mathbf{c}}_{l}+2 m[\omega]_{\times} R \dot{\mathbf{c}}_{l}+m[\omega]_{\times}^{2} R \mathbf{c}_{l}\right.\right. \\
& \left.\left.+g m \mathbf{u}_{z}\right)\right)+\left[R \mathbf{c}_{l}\right]_{\times} \dot{\omega}
\end{aligned}
$$

We see in these equations that the flexibility second order dynamics depends on the current state $\mathbf{x}$ which provides contact forces and torques, and on other constants and variables: $m, I_{l}, \dot{I}_{l}$, $\mathbf{c}_{l}, \dot{\mathbf{c}}_{l}, \ddot{\mathbf{c}}_{l}, \sigma_{l}$ and $\dot{\sigma}_{l}$. All of these latter variables are expressed in the local frame and are available from the robot embedded controllers. They are considered then as known inputs to our system and we will not discuss about the way they are computed. Therefore our model describes the flexibility dynamics in a way that enables the prediction of flexibility deformation using available data at each instant.

It is worth to notice that state positions and velocities are continuous regardless the modification of the number of contacts or their positions. This is due the the definition of the flexibility as a general rigid transformation from $\mathcal{R}_{l}$ to $\mathcal{R}$. 


\section{FLEXIBILITY STATE ESTIMATION}

In this section we show how we use the presented model to observe both the flexibility state (4) and contact wrenches.

\section{A. Observability}

We aim at demonstrating the observability of the model described above using only the gyrometer and accelerometer measurements in the IMU and contact position in $\mathcal{R}_{l}$. Internal dynamics variables are directly obtained from the robot internal joint positions, velocities and accelerations. They are assumed perfectly known and do not require any additional observer.

The gyrometer measures its angular velocity $\mathbf{y}_{g}$ in $\mathcal{R}$ but expressed in the IMU reference frame. Since the kinematics of the sensor in local reference frame $\mathcal{R}_{l}$ is well known, we know $\mathbf{y}_{g, l}$ the gyrometer measurement expressed in $\mathcal{R}_{l}$. Its expression is:

$$
\mathbf{y}_{g, l}=R^{T} \omega+\omega_{g, l}
$$

where $\omega_{g, l}$ is the angular velocity of the gyrometer in $\mathcal{R}_{l}$. This expression lead us directly to the observability of $R^{T} \omega$, the angular velocity of the flexibility expressed in $\mathcal{R}_{l}$. By derivation, the observability of $R^{T} \dot{\omega}$ is guaranteed.

The accelerometer measures $\mathbf{y}_{a}$, the sum of its own acceleration $\ddot{\mathbf{p}}_{a}$ in $\mathcal{R}$ with the gravitational field both expressed in the IMU frame. Using the known kinematics of the IMU in $\mathcal{R}_{l}$, the accelerometer measurement $\mathbf{y}_{a, l}$ expressed in $\mathcal{R}_{l}$ is then:

$$
\mathbf{y}_{a, l}=R^{T}\left(\frac{d^{2}}{d t^{2}}\left(R \mathbf{p}_{a, l}+\mathbf{t}\right)+g \mathbf{u}_{z}\right)
$$

with $\mathbf{p}_{a, l}$ and $R_{a, l}$ known values representing respectively the position and the orientation of the accelerometer in $\mathcal{R}_{l}$. By derivation:

$$
\begin{aligned}
\mathbf{y}_{a, l}= & {\left[R^{T} \dot{\omega}\right]_{\times} \mathbf{p}_{a, l}+\left[R^{T} \omega\right]_{\times}^{2} \mathbf{p}_{a, l}+2\left[R^{T} \omega\right]_{\times} \dot{\mathbf{p}}_{a, l} } \\
& +\ddot{\mathbf{p}}_{a, l}+R^{T} \ddot{\mathbf{t}}+R^{T} g \mathbf{u}_{z}
\end{aligned}
$$

Using the observability of $R^{T} \omega$ and $R^{T} \dot{\omega}$ and the known kinematic of the IMU we obtain the observability of the following part of the equation:

$$
R^{T} \ddot{\mathbf{t}}+R^{T} g \mathbf{u}_{z}
$$

The goal is then to demonstrate that our second order dynamical model used with given measurements permit to decouple the two terms of expression (29). We rewrite (16) as:

$$
\begin{aligned}
R^{T} \mathbf{F}_{c}= & {\left[R^{T} \dot{\omega}\right]_{\times} m \mathbf{c}_{l}+\left[R^{T} \omega\right]_{\times}^{2} m \mathbf{c}_{l}+2 m\left[R^{T} \omega\right]_{\times} \dot{\mathbf{c}}_{l} } \\
& +m \ddot{\mathbf{c}}_{l}+m R^{T} \ddot{\mathbf{t}}+g m R^{T} \mathbf{u}_{z},
\end{aligned}
$$

giving us to the observability of $R^{T} \mathbf{F}_{c}$, the contact forces expressed in $\mathcal{R}_{l}$. Similarly we rewrite (23) as:

$$
\begin{aligned}
R^{T} \mathbf{T}_{c}= & {\left[R^{T} \omega\right]_{\times} I_{l} R^{T} \omega+\dot{I}_{l} R^{T} \omega+I_{l} R^{T} \dot{\omega}+\left[R^{T} \omega\right]_{\times} \sigma_{l} } \\
& +\dot{\sigma}_{l}+\left[R^{T} \mathbf{t}\right]_{\times} R^{T} \mathbf{F}_{c} \\
& +m\left[\mathbf{c}_{l}\right]_{\times}\left(R^{T} \ddot{\mathbf{t}}+g R^{T} \mathbf{u}_{z}\right)
\end{aligned}
$$

which can give us the observability of $R^{T} \mathbf{T}_{c}$, the contact torques expressed in $\mathcal{R}_{l}$, but only if $R^{T} \mathbf{t}$ is observable.

In order to simplify the study of the the observability of $R^{T} \mathbf{t}$ we consider the case a simplified contact model where the support contact is reduced to a single isotrope spring contact. We rewrite (10) as:

$$
R^{T} \mathbf{F}_{c}=-R^{T} K_{F s} \mathbf{t}
$$

which gives us directly the observability of $R^{T} \mathbf{t}$ leading to the observability of $R^{T} \mathbf{T}_{c}$. Using the same simplified assumptions we rewrite (12) as:

$$
R^{T} \mathbf{T}_{c}=-R^{T} K_{T s} \boldsymbol{\Omega}+\left[R^{T} \mathbf{t}\right]_{\times} R^{T} \mathbf{F}_{c}
$$

which gives us that $R^{T} \Omega$ is observable leading to the observability of $\boldsymbol{\Omega}$. By derivation all the rotational dynamics of the flexibility is observable. Then the observability of $R^{T} \mathbf{t}$ from (32) gives us the observability of $\mathbf{t}$ and so by derivation all the translational dynamics of the flexibility is observable. Consequently all the flexibility state dynamics is observable. Therefore, the contact forces and torques are also fully observable.

This development on a simplified model with only one spring contact can be generalized easily to the general model of (16) and (23). Since $R^{T} \mathbf{F}_{c}$ and $R^{T} \mathbf{T}_{c}$ are observable their derivatives are observable too. By recursive reasoning we can find that a model with a damping is observable too. Moreover, since the number of contacts $n_{c}$ does neither add any variable nor remove any equation the observability is still guaranteed with two or more contacts.

To conclude this study we can say that the observability of the system is guaranteed by a kinematic coupling similarly to what was presented in our previous work [9] where the coupling came from a pendulum model. However, on the contrary of the former model, our coupling lies between flexibility positions and accelerations using the contact wrenches model. In addition, the use of our model gives us the observability of yaw axis orientation with only one contact, which was not the case in our previous work.

\section{B. Extended Kalman Filtering}

We use the dynamics presented in Eq.(10, 12, 24, 25) as a state dynamical model for the prediction in an Extended Kalman Filtering. The input of the system is composed of values expressed in $\mathcal{R}_{l}$ and are given by the controller of the robot: $\mathbf{c}_{l}$ the center of mass of the robot in the local frame and its derivatives $\dot{\mathbf{c}}_{l}$ and $\ddot{\mathbf{c}}_{l}, I_{c, l}, \dot{I}_{c, l}, \sigma_{c, l}$ and $\dot{\sigma}_{c, l}$ the inertial values of the robot. The measurement vector corrects the prediction and compensates for modeling errors.

As we show in the next section, we are able with this observer to estimate both the flexibility state and the contact wrenches. Then we use them to reconstruct respectively the floating base kinematic and the CoP of the robot.

\section{EXPERIMENTS}

\section{A. Experiments and demonstrations}

For testing the performances of the observer described in III$\mathrm{B}$ we perform two experiments. (i) The first experiment is the comparison of the $\mathrm{CoP}$ (or ZMP) reconstructed from the contact wrenches estimation with the $\mathrm{CoP}$ reconstructed from force sensors. This enables to validate the CoP estimation from the wrench model. (ii) The second experiment is to compensate the hand displacements due to flexibility deformation in order to keep their position constant in $\mathcal{R}$ (Fig. 3). As described in our previous work [9] this demonstration enables to validates the estimation of 


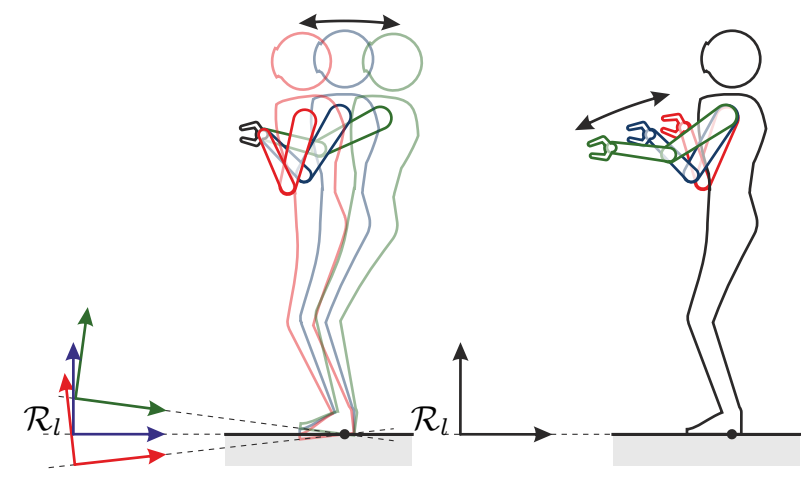

Fig. 3. Hands compensation experiment. Left: hands position is constant in $\mathcal{R}$ whereas the floating-base moves. Right: hands position moves in $\mathcal{R}_{l}$ whereas the floating-base is fix.

the floating-base kinematics: the flexibility is well estimated if the hands have constant positions and orientations in $\mathcal{R}$ despite the deformations induced by the flexible part of the robot.

Therefore, these two experiments enable to validate the performances of our observer in flexibility position/velocity with the hands compensation and acceleration with $\mathrm{CoP}$ estimation.

\section{B. Experimental settings}

The humanoid used for our experiments is HRP-2 controlled at $200 \mathrm{~Hz}$, and the sensors are the original 10 years old IMUs of the robot. Our control environment is the Stack of Tasks framework [11] which is a task-based hierarchical inverse kinematics solver. The stack of tasks gives us inputs of our model described in III-B. For the wrench model we use isotropic matrices $K_{T s}=$ $k_{t s} \mathbb{I}_{33}, K_{T d}=k_{t d} \mathbb{I}_{33}, K_{F s}=k_{f s} \mathbb{I}_{33}$ and $K_{F d}=k_{f d} \mathbb{I}_{33}$ with

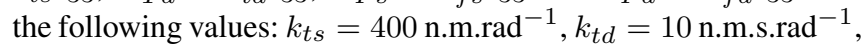
$k_{f s}=40000$ n.m ${ }^{-1}$ and $k_{f d}=600$ n.s.m ${ }^{-1}$.

$k_{t s}$ and $k_{t d}$ are average parameters identified for HRP-2 Promet-10 [12]. $k_{f s}$ and $k_{f d}$ are reasonable values which enable to simulate stiff contacts $\left(k_{f s}\right.$ high) but with stable dynamics $\left(k_{f d}\right.$ small but non zero).

The tasks tracked by the solver are set to keep both feet on the ground and the center of mass above the center position of the support polygon. For the hand compensation experiment a task was added to maintain constant position of the hands in $\mathcal{R}$ [9].

With this experimental settings we test two modes of the observer for both experiments. (i) We use the observer without any sensor feedback or correction in order to show the quality of response of our pure model. Since external disturbances cannot be detected, the perturbations are performed with a step on the reference position of the center of mass. (ii) The second mode is the observer with the feedback of sensors to show the performances of the whole observer. Perturbations are then performed by pushing the robot to excite flexibility.

\section{Results}

1) $\mathrm{CoP}$ reconstruction without sensors feedback: we give a forward step of $2 \mathrm{~cm}$ in the reference position of the center of mass. Results are shown in figure 4 . We can see that even without feedback sensors which would compensate for modeling errors we can reconstruct the position of the $\mathrm{CoP}$ with a good approximation. We can notice a bias of $3.7 \mathrm{~cm}$ between the two steady state on the

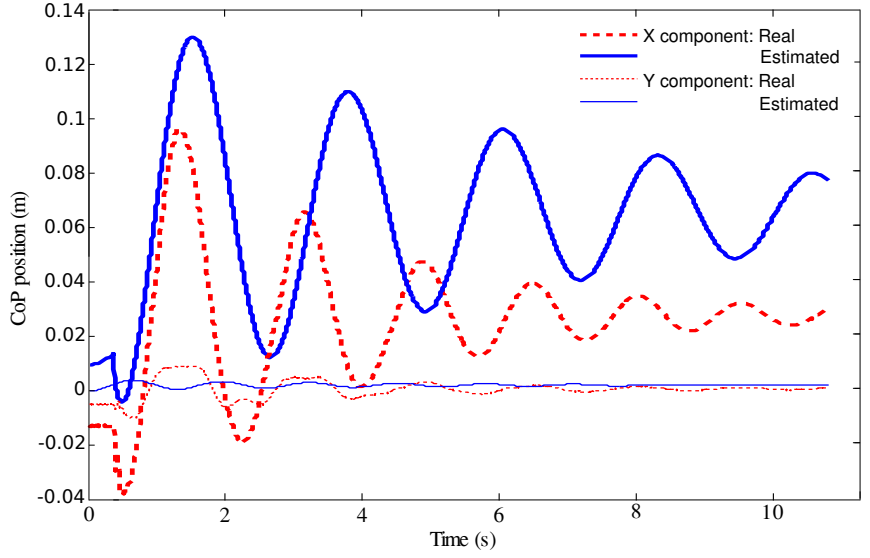

Fig. 4. Without sensors feedback for $2 \mathrm{~cm}$ forward step reference on the CoM: in blue the CoP reconstructed from our estimation of contact forces and in dashed red the real CoP reconstructed from force sensors.

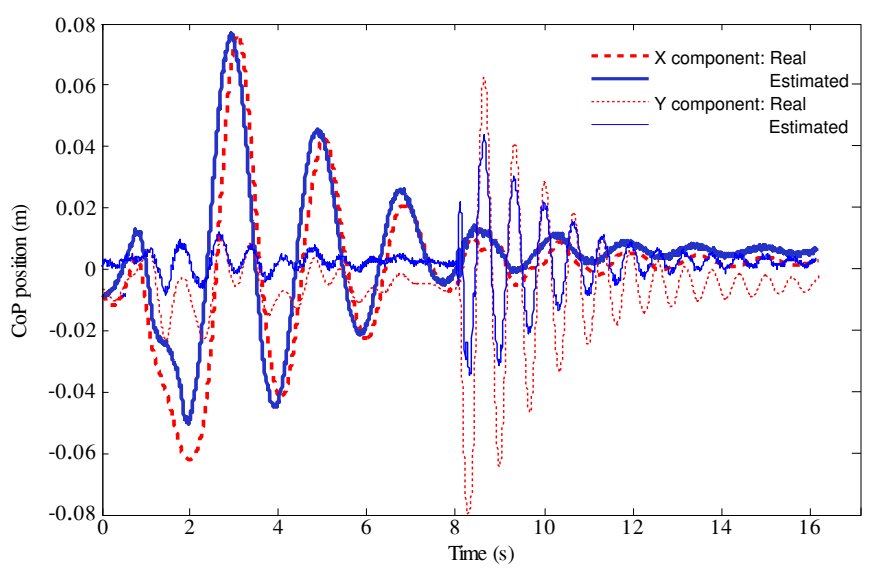

Fig. 5. With sensors feedback: in blue the CoP reconstructed from our estimation of contact forces and in dashed red the real CoP reconstructed from force sensors.

$\mathrm{x}$ component. This bias is related to the values giving by the Stack of Tasks which gathers kinematic and dynamic modeling errors.

2) CoP reconstruction with sensors feedback: we successively push the robot to excite the flexibility in rotation around the pitch and roll axis. Results are shown in figure 5. We can see that the $\mathrm{CoP}$ reconstructed during the excitation around the pitch axis is very close to the one reconstructed from force sensors. First oscillation is less well estimated because of the not modeled force of the hand pushing the robot. Contrariwise, the rotations around roll axis gives a bit poorer results due to the speed of the real dynamics and the higher stiffness of the contacts since the perturbation occurs sideways and the robot is standing on both feet.

3) Floating-base kinematic estimation: we made the hands compensation experiment setting a step reference of center of mass with and without sensors feedback. A third experiment was done with sensors feedback and pushing the robot. The performances are similar to our previous study [9]. The hand position is at 1.1 $\mathrm{m}$ distance to the contact point. If it was not compensated it would then move by about $20 \mathrm{~cm}$. Instead the hand moves by less than $2.0 \mathrm{~cm}$. The experiment is shown in the attached video. 


\section{DISCUSSION AND CONCLUSION}

We have seen how a simple model of the elasticity of the robot flexible parts enables to deduce the forces applied by the robot on the ground simply from its kinematics. This can be seen as transforming the whole robot with its IMU and flexibility into a giant force-torque sensor. Indeed, most force sensors actually detect a geometry deformation and convert it into forces and torques through an elasticity model (e.g. MEMS sensors [13]).

Since no material is perfectly stiff, every robot undergoes deformation when subject to forces and moments. The ability to extract these forces from the deformation relies on the accuracy of the kinematic reconstruction. For this reason, we had to take particular care of the quality of the flexibility observation. We have shown in our previous work the benefits of taking into account contact position constraints in the observation of the floating base kinematics. On the one hand it significantly changes the observability of the system. On the other hand it dramatically improves estimation accuracy, especially for velocities and accelerations [9].

To our knowledge the only other work integrating contact positions in kinematics observation for a humanoid robot is the work by Rotella et al [14]. They integrate directly the measurements provided by the IMU, and correct this integration by considering a model of contact with the floor. Their framework is capable to produce accurate estimations of the orientation and velocity of the robot, and their model is suitable for slippery contacts. However, the fact that they integrate directly IMU signals makes it difficult to introduce a model of the kinematics as we do by using Newton/Euler dynamics as a predictor of our observer. Also, the slippery contact model is not suitable to transform the deformation into forces and moments as we do with our spring-damper model. Therefore, this method cannot be used directly to estimate the forces at contacts. And even if the elasticity model is introduced to their solution the estimation will still lack second order precision because the forces are not used in the observer's prediction.

Indeed, the use of Newton/Euler dynamics increases the accuracy of the kinematics prediction. It even gives a direct insight to clean estimates of linear and angular accelerations which are poorly provided by accelerometers and gyrometers. Since the prediction is much more reliable, the sensor corrections are much finer and the final estimate more precise.

However, the estimation of contact forces depend also on a good estimation of visco-elastic parameters of contacts. The model of elasticity or viscosity can be wrong, for example when the identification is not done properly or when there are compliant surfaces in addition to flexibility in the robot. In that case, the estimation of the forces may be wrong. It has to be corrected using online identification techniques or by using force sensors if available. Nevertheless, even if the contact forces are wrong the correction made by the inertial sensors enable to correctly estimate the state of the flexibility, we have shown in a prior work that the worst possible model, i.e. a simple acceleration double integrator, can still be used by a state observer and the kinematic coupling due to contact forces is sufficient to guarantee a precise estimation.

It is worth to remind that this observer is intended to be used in a closed-loop whole body control in order to guarantee balance and compensation for flexibility deformations, similarly to what we did with a former model-free observer [15]. Therefore, we are currently investigating the performances of humanoid stabilizer which can dispense with force sensors and is able to absorb perturbations and correct deviations of the Center of Mass.
Finally, until now, we suggested to not use the force sensors because of their sensitivity and expensiveness. However, many robots do have reliable force sensors which are successfully used to make robots accomplish many tasks including locomotion. These sensors may provide precious corrections to forces reconstructed from our observer. Furthermore, these measurements are very easy to introduce in our observer and will potentially provide an even better estimation of the CoP than the force sensors or the presented observer separately. This will be also a subject for our next researches.

\section{REFERENCES}

[1] P.-B. Wieber. On the stability of walking systems. In Proceedings of the International Workshop on Humanoid and Human Friendly Robotics, Tsukuba, Japan, 2002.

[2] S. Kajita, M. Morisawa, K. Miura, S. Nakaoka, K. Harada, K. Kaneko, F. Kanehiro, and K. Yokoi. Biped walking stabilization based on linear inverted pendulum tracking. In Intelligent Robots and Systems (IROS), pages 4489-4496, Oct 2010.

[3] C. Ott, M.A. Roa, and G. Hirzinger. Posture and balance control for biped robots based on contact force optimization. In Humanoid Robots (Humanoids), 2011 11th IEEE-RAS International Conference on, pages 26-33, Oct 2011.

[4] S. Kajita, F. Kanehiro, K. Kaneko, K. Fujiwara, K. Harada, K. Yokoi, and $\mathrm{H}$. Hirukawa. Biped walking pattern generation by using preview control of zero-moment point. In International Conference on Robotics and Automation (ICRA), volume 2, pages 1620 - 1626, sept. 2003.

[5] N. Kanehira, T. Kawasaki, S. Ohta, T. Ismumi, T. Kawada, F. Kanehiro, S. Kajita, and K. Kaneko. Design and experiments of advanced leg module (hrp-2l) for humanoid robot (hrp-2) development. In Intelligent Robots and Systems, volume 3, pages 2455-2460, 2002.

[6] S. Kajita, K. Yokoi, M. Saigo, and K. Tanie. Balancing a humanoid robot using backdrive concerned torque control and direct angular momentum feedback. In ICRA, 2001.

[7] S. Kajita, T. Nagasaki, K. Kaneko, K. Yokoi, and K. Tanie. A running controller of humanoid biped hrp-2lr. In Intl. Conf. Robotics and Automation, (ICRA)., pages 616-622. IEEE, 2005.

[8] Aldebaran Robotics. Romeo. http://www.projetromeo.com, 2011.

[9] M. Benallegue and F. Lamiraux. Humanoid Flexibility Deformation Can Be Efficiently Estimated Using Only Inertial Measurement Units and Contact Information. In IEEE-RAS International Conference on Humanoid Robots, November 2014.

[10] Fabrizio Vecchi, Cinzia Freschi, Silvestro Micera, Angelo M Sabatini, Paolo Dario, Rinaldo Sacchetti, et al. Experimental evaluation of two commercial force sensors for applications in biomechanics and motor control. In 5th Ann. Conf. Int. Functional Electr. Stimulation, 2000.

[11] N. Mansard, O. Stasse, P. Evrard, and A Kheddar. A versatile generalized inverted kinematics implementation for collaborative working humanoid robots: The stack of tasks. In International Conference on Advanced Robotics, pages 1-6, June 2009.

[12] Y. Mikami, T. Moulard, E. Yoshida, and G. Venture. Identification of hrp-2 foot's dynamics. In Intelligent Robots and Systems (IROS 2014), 2014 IEEE/RSJ International Conference on, pages 927-932, Sept 2014.

[13] F. Beyeler, S. Muntwyler, and B.J. Nelson. A six-axis mems forcetorque sensor with micro-newton and nano-newtonmeter resolution. Microelectromechanical Systems, Journal of, 18(2):433-441, April 2009.

[14] Nicholas Rotella, Michael Bloesch, Ludovic Righetti, and Stefan Schaal. State estimation for a humanoid robot. In Intelligent Robots and Systems (IROS 2014), 2014 IEEE/RSJ International Conference on, pages 952-958. IEEE, 2014.

[15] Mehdi Benallegue and Florent Lamiraux. Estimation and stabilization of humanoid flexibility deformation using only inertial measurement units and contact information. International Journal of Humanoid Robotics, 13(3):1550025-1 to 1550025-20, 2015. 